\title{
MedienPädagogik
}

Zeitschrift für Theorie und Praxis der Medienbildung

\section{Offene Bildungspraxis erlebbar machen - die Rolle von Podcasts für Projektdokumentationen und Re- flexionsprozesse}

Constanze Reder und Bence Lukács

\begin{abstract}
Zusammenfassung
Der vorliegende Beitrag widmet sich der Frage, wie Reflexion als Teil akademischer Medienkompetenz an Hochschulen adressiert werden kann. Als Beispiel dient ein Praxisprojekt, über das Studierende in labs mit Open Educational Resources (frei verfügbares Bildungsmaterial, OER), aber darüber hinaus vor allem mit Open Educational Practices (offenen Bildungspraktiken, OEP) und offenen Lehrinhalten in Kontakt kommen. Dabei stehen auch andere hochschulische Akteursgruppen im Fokus, die gemeinsam mit den Studierenden in moderierten Dialogen darüber beraten, wie nicht nur OER sondern auch OEP an der Hochschule etabliert werden können, um Dozierenden wie Studierenden den Zugang zu offener Bildung zu erleichtern. Ausgehend von dieser Praxiserfahrung greift der Artikel den Baustein des projektbegleitenden Podcasts heraus, der die Anlage des Projekts dokumentiert, aber auch ein reflexives Element im Doing darstellt, durch das die verschiedenen Perspektiven der Mitarbeitenden an den beiden Standorten, aber auch die von Stakeholdern und Kooperationsakteurinnen und Kooperationsakteuren widergespiegelt wird. Er widmet sich der Frage, wie dementsprechend ein reflektierter Umgang mit offener Lehr-Lernpraxis an der Hochschule unterstützt werden kann und wie offene Praxis nicht nur als Seminarinhalt, sondern auch nachhaltig als Teil von Hochschulentwicklungsprojekten implementiert werden kann.
\end{abstract}

How to experience Open Educational Practices - the role of Podcasts in documentary and reflective processes of universary projects

\begin{abstract}
This paper deals with the question of how reflection as part of academic media literacy can be addressed at universities. An example of this is a practical project, where students in labs come into contact with Open Educational Resources (OER), but above all with Open Educational Practices (OEP) and open teaching material. There is also focus on other relevant university actors, which together with the students discuss how not only OER, but more importantly OEP in general can be established at the university, in order to facilitate access to open education for lecturers and learners alike. On the basis of this practical experience, the article outlines the projects podcast that works as an instrument of
\end{abstract}


documentation and also as a channel, wherein project staff at both universities, as well as other university stakeholder can openly reflect their experiences. The question addressed relates to which project elements can be supported with a reflective approach regarding open-learning and-teaching practice at the university, and how Open Educational Practices can be more than content for lectures, and instead sustainably be implemented as part of developmental higher education projects.

OERlabs: Zwischen labs, Multistakeholder-Dialog und dem Bildungsshaker

Im Kern des Verbundprojekts «OERlabs - (Lehramts-)Studierende gemeinsam für OER ausbilden» der Universität zu Köln und der Technischen Universität Kaiserslautern geht es darum, sich im sich im Laborformat mit OER auseinanderzusetzen. Zusätzlich zu den labs, in denen gestaltet und experimentiert werden soll, sind an beiden Hochschulen Multistakeholder-Dialoge (MSD) implementiert worden, die an Runden Tischen solchen Akteursgruppen Möglichkeit zum Austausch bieten, die sich sonst eher selten begegnen ${ }^{1}$. Um die Erfahrungen und entstandenen Projektschritte mit den jeweiligen Zielgruppen auszutauschen, wurde im Projekt der Podcast Bildungsshaker eingeführt. Der Bildungsshaker ist ein reflexives Element in der Projektdokumentation. Neben der Aufgabe, Informationen unter den Beteiligten auszutauschen, dient er dazu, die einzelnen Projektschritte und deren Entstehung zu dokumentieren und das Vorgehen zu reflektieren. Es lohnt sich demnach, inn näher zu betrachten. Im folgenden Beitrag werden wir zunächst auf den Einsatz von Podcasts in der Hochschulentwicklung und der Projektdokumentation allgemein eingehen und den Bildungsshaker insbesondere vorstellen. Davon ausgehend untersuchen wir, inwiefern Podcasts als Instrument zur Reflexion eingesetzt werden können und wie der hier entstehende Mehrwert für die Förderung von Medienkompetenz an Hochschulen nutzbar gemacht werden kann.

Das Ziel ist, so schliesslich Reflexion, hochschulische Medienkompetenzförderung und Projektdokumentation über das Element des Bildungsshakers zusammenzuführen.

1 Studierende, Lehrende, Akteurinnen und Akteure aus der Medien-, Hochschul-, und Schulpraxis kommen in den labs auf Augenhöhe zusammen, um OER kennenzulernen, selbst zu produzieren und zu teilen. Sie lösen sich dabei vom Besucherinnen- oder Besucher- bzw. Teilnehmenden-Status und lernen mit- und voneinander. Dazu werden viele Methoden der Aktivierung und Vernetzung an den beiden Hochschulen entwickelt und getestet. In den MSD zum Beispiel tauschen sich Prorektorinnen und Prektore für Studium und Lehre mit Mitarbeitenden hochschulischer Bildungseinrichtungen sowie regionalen Bildungsträgern aus der Lehrpersonenbildung zu OER, offener Bildung und offener Lehre aus. Diese MSD finden mehrfach während der Projektlaufzeit statt. 
Podcasting als vielfältiges Instrument in der Hochschulentwicklung

Podcasts erfreuen sich seit einigen Jahren grosser Beliebtheit und sind Produzentinnen und Produzenten und Nutzerinnen und Nutzern zumeist durch die aktive Medienarbeit sowie als Bestandteil der professionellen und semiprofessionellen Radioarbeit vertraut oder aus Lehrveranstaltungsaufzeichnungen bekannt. Der Bildungsshaker-Podcast steht als Mischform zwischen diesen Verwendungszwecken. Aktuelle Untersuchungen zeigen, dass die Anzahl der Hörerinnen und Hörern weiterhin steigt und vor allem das jüngere Publikum zusehends mehr auf dieses Medium setzt. Zudem wurde deutlich, dass Nutzerinnen und Nutzern von Audio- und Videomedien auch eher bereit sind, auf dieses Medium umzusteigen, sobald sie von Podcast-Angeboten erfahren (Oswald 2018). Allerdings hatten Podcasts schon vor mehreren Jahren Einzug in die Hochschullandschaft gehalten, wo sie in unterschiedlichen Szenarien eingesetzt und verwendet wurden (und werden).

Einer der grundlegenden Verwendungen von Audiomitschnitten an der Hochschule war die Möglichkeit, Vorlesungen bzw. relevante Inhalte ausserhalb der geregelten Vorlesungs- und Seminarzeiten flexibel und dem eigenen Ermessen nach anzuhören. Chris Evans stellte 2008 in seiner Studie fest, dass Studierende die Möglichkeit, Inhalte auch unterwegs anzuhören, als sehr positiv wahrgenommen haben, sowie das Gefühl hatten, dass sie mit Hilfe von Audiomitschnitten besser lernen konnten als mit Notizen und Lehrbüchern $(2008,496)$. Lonn und Teasley (2009) berichteten von ähnlichen Ergebnissen, stellten jedoch auch die Frage, ob Podcasts tatsächlich Veränderungen in der Hochschule hervorrufen können, oder wie zuvor erwähnt, Audiomitschnitte lediglich Lehrbücher als Hauptmaterial ersetzen.

\section{Podcasting als Instrument der Projektdokumentation und Open Education}

Hinter dem Bildungsshaker-Podcast steht allerdings nicht dieser traditionelle Anspruch, Lehrveranstaltungsinhalte an Studierende zu vermitteln, sondern das Ziel, Aktivitäten innerhalb eines Projektes (bzw. der Hochschule) zu dokumentieren, zu reflektieren und der hochschulinternen wie -externen Öffentlichkeit zur Verfügung zu stellen. Er ist nicht bloss Teil von Lehr-/Lernmaterialien und doch dokumentiert er hochschulische Prozesse als Teil eines hochschulischen Projektes. Gleichzeitig ist es durch das Format des Podcasts, in dem Antworten gegeben werden können, möglich, damit einen partizipativen Charakter in den Arbeitsprozess einzubringen. Ähnliche Ziele verfolgte 2009 der Augsburger KaffeePod, in dem Hochschulangehörige die Arbeit und das Leben an der Hochschule in narrativer Form präsentierten, um vor allem externen Personen einen Einblick in das Hochschulsystem zu geben (Hofhues und Bianco 2009). Im Bildungsshaker-Podcast berichten, dokumentieren und reflektieren die wissenschaftlichen Mitarbeiterinnen und Mitarbeiter Geschehnisse aus dem Projekt. Die Entscheidung, einen projektbegleitenden Podcast zu machen, 
entstand zum einen aus diversen, im Projektantrag behandelten Konzepten (Stichworte Offenheit, Evaluation und Partizipation) sowie aus der Umsetzung reflexiver Praxis aus dem schriftlichen Portfoliobereich (vgl. Bräuer 2007).

Im Sinne der Offenheit soll mit dem Podcast der Open Gedanke nach aussen getragen werden und Gedankengänge und Arbeitsschritte «sichtbar» bzw. hörbar gemacht werden. Ideen, Planungen und Hürden werden offen thematisiert und bieten den Projektmitarbeitenden Möglichkeiten, auch kleinteilige bzw. informelle Gespräche zu kontextualisieren. Die öffentliche Reflexion in Podcast-Form bedeutet eine Beschäftigung mit einer Thematik auf einer neuen Ebene. Dies ermöglicht eine Art Monitoring, da Erkenntnisse aus den Reflexionen genutzt werden, und dadurch im Projektverlauf mögliche Veränderungen umgesetzt werden können. Beispielhaft kann hier die Reflexion über die Planung der Multistakeholder-Dialoge ${ }^{2}$ angeführt werden (Episode 9: Planung des Multistakeholder-Dialogs: Utopisches Denken), in dem sehr offen Fragen aufgeworfen wurden. Dieses offene Ansprechen von Überlegungen und etwaigen Problemen führte über die Verbreitung der Episode durch Twitter zu einem informellen Gespräch mit anderen Hochschulangehörigen, die von ihren eigenen Erfahrungen zur Problemstellung hilfreiche Hinweise geben konnten. Des Weiteren setzt der Podcast eine partizipative Ebene um, da Probleme gemeinsam bearbeitet werden und damit alle relevanten Akteuren in den Arbeitsprozess und die Gedankengänge des Projektes eingebunden werden, siehe z.B. eine Episode über das Lehramtsstudium an der Universität zu Köln (Episode 14: Wie läuft ein Lehramtsstudium in Köln ab?), sowie Episoden zum Urheberrecht und der Praxis in den OERlabs-Seminaren (Episode 15: OERlabs-Studierende über ihr Spielbrett-Projekt in Kaiserslautern).

\section{Podcasting als Instrument der Reflexion}

Die Produktion des Bildungsshaker-Podcasts stellt selbst keine Massnahme der Medienkompetenzförderung für bestimmte Zielgruppen durch aktive Medienarbeit dar. Dennoch kann ein Podcast im Kontext der akademischen Medienkompetenzförderung als sinnvolle Methode integriert werden, da er als Mittel der Kommunikation und Produktion mit verschiedenen Akteursgruppen der Hochschule dient. Bezüge zwischen dem Bildungsshaker-Podcast der OERlabs und den Bereichen der akademischen Medienkompetenzförderung finden sich bei Wedekinds Forderungen an die nachhaltige und erfolgreiche Integration digitaler Medien in die akademische Lehre: Er plädiert einerseits für die Unterstützung konkreter Umsetzungen vor Ort und andererseits für eine Kultur des Austausches und der Kollaboration durch Open Source,

2 In den Dialog-Veranstaltungen kommen mehrere Stakeholder (Interessenvertreterinnen und Interessenvertreter) zusammen, um gemeinsam Themen- oder Fragestellungen zu bearbeiten. Die eingeladenen Personen bringen hierbei ihre vielfältigen Perspektiven mit in den Dialog ein, treten aber auch in den offenen Austausch mit- und untereinander. 
Open Access und Open Content (vgl. 2009, 11f.). Beides findet sich im Bildungsshaker-Podcast wieder, zum einen durch die inhaltlichen Bemühungen, nicht nur Ergebnisse eines Projekts, sondern auch einzelne Schritte sichtbar zu machen und so anderen Projekten als Anhaltspunkt zu dienen. Und zum anderen fördert der Podcast die Zusammenarbeit an Hochschulen durch die Partizipation unterschiedlicher Hochschulangehöriger, zum Teil sogar verschiedener Universitäten. Zusätzlich wird offene Praxis durch die CC-BY-Lizenz ${ }^{3}$ der einzelnen Folgen ausgeübt. Ziel ist es, das Reflektieren von Projektschritten als Schnittstelle von Medienkompetenzförderung an Hochschulen und offener (Projekt)-Praxis zu ermöglichen.

Obwohl die reflexive Praxis im Hochschulkontext in den letzten Jahren ihren Schwerpunkt auf die Ausarbeitung von Portfolios - also eine schriftliche ReflexionsPraxis - legt, lässt sich dieses Konzept im Bildungsshaker-Podcast auch in mündlicher Form entsprechend umsetzen. Bräuer (2007) beschrieb die reflexive Praxis der sprachlichen Handlung in drei Ebenen, denen hier jeweils Beispiele aus der Bildungsshaker-Podcast-Praxis zugeordnet werden:

- Auf der ersten Ebene Dokumentieren und Beschreiben werden zunächst relevante Informationen gesammelt und geordnet. Im Bildungsshaker-Podcast manifestierte sich dieser Schritt in den ersten Episoden, in denen zunächst das Projekt beschrieben wurde (Episode 1: Über die OERlabs), sowie Einblicke in die Thematik OER gegeben wurden (Episode 5: Eine Reise durch die OER-Projektwelt).

- Die folgende Ebene, Analysieren und Interpretieren, beschreibt Bräuer $(2007,1)$ als die Weiterführung der ersten Ebene da das Dokumentierte und Beschriebene einer Qualitätssicherung unterzogen wird und die Projektmitarbeitenden sich ihres Handelns bewusst werden können. Die gewonnenen Erkenntnisse können das neu erworbene Wissen mit bereits vorhandenen Erfahrungen verknüpfen, und dadurch neue Einsichten erzielen (z.B. die Beschreibungen und Verarbeitung der MSD-Events an den jeweiligen Standorten Episode 10: Der MultistakeholderDialog aus Sicht der TU Kaiserslautern und Episode 12: We have a Lift-Off (MSD1 Köln)).

- Auf der letzten Ebene soll das Gelernte mit den Zielen abgeglichen werden, und dementsprechend anhand der gesetzten Erwartungen evaluiert werden. An dieser Stelle kommt erneut der Faktor Monitoring ins Spiel, da letztendlich der Projektantrag mit den Projektergebnissen unter Zuhilfenahme der Podcast-Reflexionen verglichen werden können.

3 Neben der bekannten urheberrechtlich bedingten Einschränkung «Alle Rechte vorbehalten», bietet eine gemeinnützige Gesellschaft namens Creative Commons (CC) online verschiedene Lizenzverträge an. Mittels dieser Lizenzen können Urheberinnen und Urheber von Werken und Medien einer Person oder auch der Öffentlichkeit verschiedene Nutzungsrechte einräumen. Die Lizenzen definieren unterschiedliche Rechte, bspw. Weitergabe unter gleicher Lizenz oder keine Erlaubnis zu Veränderung, und können auch kombiniert werden. Die CC-BY Lizenz erlaubt anderen, das betreffende Werk zu verbreiten, zu remixen, also zu bearbeiten und auch kommerziell zu nutzen, solange Sie als Urheber des Originals genannt werden. Sie trägt zur maximalen Verbreitungs- und Nutzungsmöglichkeit des Werkes bei. 
Zudem kann man den Podcast auch als Reflexionsinstrument der Projektmitarbeitenden sehen. Den meisten der zahlreichen Reflexionskonzepte der letzten Jahrzehnte ist gemeinsam, dass es einen bestimmten Anlass, einen Auslöser geben muss, damit Reflexionsprozesse überhaupt ablaufen, geschweige denn gelenkt werden können ${ }^{4}$. Je nach Ansatz, Zeit und Konzept können das ein Gefühl, eine Empfindung sein (Gibbs 1988), eine prägende Erfahrung (Kolb 1984) oder ein Misserfolg (Dewey [1910]1951). Grundsätzlich entwickelten sich Reflexionsmodelle, wie der «reflective cycle» von Gibbs (1988), aus den Ideen und Modellen des Lernens heraus, Stichwort ist hier stellvertretend der «learning cycle» von Dewey. Hilzensauer zielt in einem Diskussionsbeitrag von 2008 darauf ab, diese Konzepte um die unterschiedlichen Ebenen der Reflexion zu ergänzen. Er bedient sich dazu der Dialogischen Didaktik, um die wesentlichen Elemente der Reflexion und die Reflexion für das Lernen selbst dabei expliziter zu beschreiben (vgl. Hilzensauer 2008, 11f.). Die Dialogische Didaktik wurde von Urs Ruf gemeinsam mit Peter Gallin entwickelt und bezeichnet ursprünglich ein dreischrittiges Lehrmodell (vgl. Ruf 2008, 13f.) bei dem man mit sich und anderen in einen Dialog tritt, der von den Leitfragen «lch mache das so (1), Wie machst du es? (2), Das machen wir ab (3)» unterstützt wird (Gallin und Ruf 1995, zitiert nach Badr-Goetz 2007, 45). Der Mehrwert des Modells für die Reflexionskompetenz liegt in diesem Perspektivwechsel (vgl. Hilzensauer 2008, 11). Der Lernkontext bleibt so kein nach innen geschlossener und verengter, sondern öffnet sich für Vergleiche und andere Handlungsoptionen. Diese Erweiterung der Reflexionsebenen um den wichtigen Aspekt der gemeinsamen Reflexion als Gespräch, als Dialog, rahmt auch den Ansatz für den Bildungsshaker-Podcast. Auch dieser eröffnet durch das Offenlegen und Diskutieren über das eigene Projektschaffen sowohl den Erzählenden, den Diskutanten, aber auch den Zuhörenden neue Wege für weiterführende Handlungsoptionen.

\section{Adressierung akademischer Medienkompetenz}

Blickt man mit diesem Erkenntnisstand auf die Perspektive um offene Bildungspraxis und Hochschulentwicklung, unter Rekursion auf digitale Medien an der Hochschule, so können Bezüge hergestellt werden zum Konzept akademischer Medienkompetenz. Akademische Medienkompetenz orientiert sich vor allem an der genauen Definition ihrer Zielgruppen und der akademischen Arbeitsbereiche. So werden neben den Hochschullehrenden und sonstigen Hochschulmitarbeitenden auch Studierende einbezogen (vgl. Reinmann, Hartung, und Florian 2013, 2), selbst wenn diese «eine andere Mediensozialisation und daraus resultierende Nutzungsgewohnheiten entwickelt haben» (Wedekind 2009, 1). Zusätzlich fällt der Blick auf die akademischen Arbeitsbereiche der Lehre, Forschung, Anwendung und (Selbst-)Verwaltung (Reinmann

4 Weitere Ausführungen zu Reflexionskonzepten und Zusammenfassungen auch zu finden bei Glade, Reder, und Schiefner-Rohs 2017, 67-80. 
et al. 2013) sowie die akademischen Tätigkeiten, denen Studierende an der Hochschule nachgehen. Ausgehend von dieser Annahme orientieren sich entsprechende Medienkompetenzen an der Hochschule an dem Bedarf, was zur Erledigung und Erfüllung des jeweiligen Arbeitsbereichs notwendig wird. Folgerichtig konstatiert auch Wedekind, dass im Vergleich zu ausseruniversitären Medienbildungs- und medienpädagogischer Konzepte an der Universität die «instrumentell-technische Komponente» $(2009,2)$ gegenüber den pädagogischen Absichten der Medienkompetenzförderung an Bedeutung gewinnt. So stehen die Ausgestaltung des Arbeitsbereichs mit digitalen Medien, beispielsweise von Lehr- Lernumgebungen und Verwaltungsorganisation, und die dazu nötigen technischen Fertigkeiten der Mitarbeitenden, im Vordergrund.

Eine genauere Vorstellung davon, in welchen dieser hochschulischen Arbeitsbereiche welche Medienkompetenzen gebraucht werden, wird bei Reinmann et al. vermittelt. Im Wesentlichen orientieren sich die drei grossen Arbeitsbereiche und die Zuteilung der erforderlichen Kompetenzen an der Kategorisierung von Hochschulmitarbeitenden (vgl. Reinmann et al. 2013, 3ff.).

\begin{tabular}{|l|l|}
\hline Lehre und Lernen & $\begin{array}{l}\text { Hier geht es um Medienkompetenz als mediendidaktische Kompetenz, } \\
\text { die Lehrende befähigt, Lehrszenarien mit digitalen Medien zu gestal- } \\
\text { ten und als aktivierende Anforderung an Studierende, sich mit Medien } \\
\text { Wissen im Selbststudium anzueignen und so bestimmte Fähigkeiten } \\
\text { weiterzuentwickeln. }\end{array}$ \\
\hline $\begin{array}{l}\text { Forschung und } \\
\text { wissenschaftliches }\end{array}$ & $\begin{array}{l}\text { Medienkompetenz von Forschenden wird hier zur Bewältigung speziell } \\
\text { der Forschung zugeordneter Aufgaben erforderlich: Einwerben von } \\
\text { Drittmitteln, Recherchekompetenz, Forschungsdatenmanagement, } \\
\text { zeitgemässes Publizieren sowie Kommunikation, Vernetzen und aktives } \\
\text { Produzieren mit- und untereinander. }\end{array}$ \\
\hline $\begin{array}{l}\text { Selbstverwaltung } \\
\text { und Studiumsorga- } \\
\text { nisation }\end{array}$ & $\begin{array}{l}\text { Den digitalen Verwaltungsabläufen anderer Organisationen ähnlich, } \\
\text { kommen bei der akademischen Selbstverwaltung das Management } \\
\text { ganzer Module, Studiengänge und Veranstaltungen sowie das Manage- } \\
\text { ment von Gremiensitzungen und der anschliessende Informationsfluss } \\
\text { mit Hilfe digitaler Medien hinzu. Bedienungs- und Technikkompetenz } \\
\text { sind dazu erforderlich. }\end{array}$ \\
\hline
\end{tabular}

Tab. 1.: Hochschulische Arbeitsbereiche und dort erforderliche Medienkompetenzen.

Durch die in der Tabelle 1 dargelegten Aufgaben der jeweiligen Mitarbeitenden wird der Fokus auf technische und organisationale Medienkompetenzen an einer Hochschule verständlicher. Die Weiterentwicklung anderer Medienkompetenzen hängt vom Bedarf im Alltag ab, zudem werden die verschiedenen Tätigkeitsfelder selten miteinander in Kontakt gebracht. Somit ist es auch Aufgabe hochschulischer Medienbildung Medienkompetenzen, die hier vor allem den sicheren Umgang und den sinnvollen Einsatz digitaler Medien in den jeweiligen Arbeitsprozessen meinen, umfassend in den akademischen Arbeitsbereichen zu fördern. 
Inwiefern heute Lehrende an Hochschulen an Massnahmen zur Förderung ihrer Medienkompetenzen teilnehmen, lässt sich nach den älteren Untersuchungen von Wedekind und Reinmann et al. nicht abschliessend klären. ${ }^{5}$ Es lassen sich allerdings viele Bestrebungen identifizieren, Medienbildungsangebote an Hochschulen voran zu treiben, die auf einen signifikanten Bedarf in diesem Bereich hinweisen. So diskutiert unter anderem das Hochschulforum Digitalisierung die Einflüsse der Digitalisierung auf die Hochschule und insbesondere auf die Hochschullehre. Die AG Digitale Medien und Hochschuldidaktik der Deutschen Gesellschaft für Hochschuldidaktik möchte die Angebotsstruktur hochschuldidaktischer Einrichtungen hinsichtlich des Einsatzes digitaler Medien weiterentwickeln, während sich die Gesellschaft für Medien in der Wissenschaft e.V. hauptsächlich um die Mediennutzung in Forschung und Lehre bemüht. Mit der Deutschen Initiative für Netzwerkinformation (DINI) werden für Forschung, Lehre, Studium und Verwaltung an Hochschulen passende IT-Unterstützungsangebote entwickelt, e-teaching.org des Leibniz-Instituts für Wissensmedien berät bei der Hochschulbildung mit digitalen Medien. Solche und ähnliche Angebote verdeutlichen den aktuellen Weiterbildungsbedarf bei der akademischen Medienkompetenz besonders.

Bleibt die Frage, ob durch den Einsatz eines Podcasts wie des Bildungsshakers Elemente der akademischen Medienkompetenzentwicklung adressiert werden können. Bei genauerer Betrachtung entsteht hier ein ambivalentes Bild, das im Folgenden mit Verweis auf das Konzept von Reinmann et al. skizziert wird.

Im betreffenden Konzept finden sich drei wichtige Verbindungspunkte. Erstens kann der Bildungsshaker-Podcast durchaus als Mittel der offen-lizenzierten, wissenschaftlichen Online-Publikation gelten, die Reinmann et al. als Teil der Medienkompetenz im Bereich Forschung betrachten (vgl. 2013, 4). Zwar werden über den Podcast keine wissenschaftlichen Erhebungsergebnisse veröffentlicht, aber er ist als Impulsgeber gedacht, wichtige Projektschritte zu analysieren und sie so darzustellen, dass Projektmitarbeiter anderer universitärer Projekte von den Erfahrungen profitieren können. Insofern steht die Reflexion von Erfahrungen im Podcast als Mittel zur Verfügung, ein bisher implizites Wissen von Mitarbeitenden, Kooperationspartnerinnen und Kooperationspartner und Studierenden zu explizieren und für andere zugänglich zu machen. So stellen zweitens auch die Kommunikation unter Wissenschaftlern und deren gemeinsame Produktivität in Communities ein Beispiel für den Austausch dar (vgl. ebd.). Und drittens schliesst sich an diesen Aspekt unmittelbar die Massnahme

5 In seiner kurzen Untersuchung, welche Massnahmen zur Förderung akademischer Medienkompetenz bei Hochschulmitarbeitenden existieren, identifiziert Wedekind angelehnt an die E-Learning-Kompetenzbereiche ein Überangebot für die Universitätsangehörigen (Wedekind 2009). Ausgehend von den bekannten Teilnehmerzahlen sei aber nur eine geringe Anzahl von Hochschullehrenden mit diesen Kursen, deren Konzept sich an den jeweiligen akademischen Arbeitsplätzen orientiert, zu erreichen (vgl. Wedekind 2009, 8). Bei Reinmann et al. scheint diese Beobachtung Bestand zu haben. Sie fordern eine umfassendere Medienkompetenzförderung, die digitale Medien in die hochschulischen Arbeitsbereiche selbstverständlich integriert und direkt an anstehende Aufgaben aus den Arbeitsbereichen anschliesst (vgl. 2013, 6). 
der Medienkompetenzförderung an Hochschulen durch Kulturentwicklung in Form der Open-Bewegung (gemeint sind OER, Open Access und Open Educational Practices) an (vgl. ebd., 8). Durch den Podcast wird nicht nur der Community, sondern auch dem «befähigten Bürger» (ebd.) grundsätzlich ermöglicht, an Entwicklungsprozessen und Projektergebnissen der Universität teilzuhaben.

Wie bereits im vorherigen Kapitel deutlich wurde, sind dem Podcast viele reflexive Elemente eigen. Reflexion als (Teil-)Kompetenz kommt im Konzept der akademischen Medienkompetenz, anders als in den meisten Modellen zu (informeller) medienpädagogischer Kompetenz ${ }^{6}$, aber kaum bzw. gar nicht vor. Gerade im Hinblick auf den Diskurs zu digitalen Medien und Hochschulentwicklungsstrategien fällt diese Leerstelle in den Konzepten besonders auf. Denn Reflexion und Medien können in vielerlei Hinsicht miteinander in Verbindung stehen.

Hinsichtlich des Bildungsshaker-Podcast-Beispiels werden vor allem zwei Ebenen dieses Verhältnisses sichtbar:

1. Medien können dazu eingesetzt werden, Reflexion erst zu ermöglichen. Sie werden so zu Instrumenten, welche sinnvolle reflexive Zugänge schaffen und verschiedene Reflexionstypen ansprechen können.

2. Ergänzend dazu steht die Reflexion über das eigene Handeln mit digitalen Medien. Anschliessend zum eigentlichen Umgang mit Medien selbst wird hier das pädagogische und didaktische Handeln im Hinblick auf Sinnhaftigkeit und kompetenten Umgang untersucht.

Reflexionen dieser Art eröffnen Mediennutzerinnen und Mediennutzer kognitive und affektive Wege, das eigene Medienhandeln in Frage zu stellen und können so zur Qualitätssicherung und Weiterentwicklung des Umgangs mit digitalen Medien an der Hochschule beitragen. Je nach akademischem Arbeitsbereich wirkt sich eine solche Reflexion auch auf didaktisch sinnvoll angelegte Lehr-Lernszenarien und den pädagogisch wertvollen Inhalt von Lehrveranstaltungen aus.

\section{Fazit}

Abschliessend lässt sich feststellen, dass diese Art von Projektdokumentation und Reflexion sich nicht für jede Art von Projekt eignet. Durch die zuvor angesprochenen Elemente (Open Gedanke, Monitoring, Partizipation) ist es für die OERlabs jedoch besonders gut geeignet, das Projekt mit einem Podcast zu begleiten. Denn in Hochschulprojekten ist eine ausführliche und begleitende Dokumentation der Projektschritte und der Arbeitsprozesse stets von grosser Bedeutung. Sie dient sowohl einer Bestätigung für etwaige Geldgeber, dass das Projektbudget gemäss dem

6 Siehe u.a. Konzepte von Baacke 1996, Blömeke 2005, Tulodziecki 2012. 
Projektantrag umgesetzt wird, als auch den durchführenden Instanzen und der relevanten Community zur Präsentation von Projektergebnissen. Der BildungsshakerPodcast bietet das Potenzial, das breite Spektrum an Akteurinnen und Akteuren miteinander (thematisch) in Kontakt zu bringen, sowie für neue Arbeits- und Denkweisen zu sensibilisieren.

\section{Literatur}

Baacke, Dieter. 1996. «Medienkompetenz - Begrifflichkeit und Sozialer Wandel». In Medienkompetenz als Schlüsselbegriff. Theorie und Praxis der Erwachsenenbildung, hrsg. v. Antje von Rein, 112-124. Bad Heilbrunn: Deutsches Institut für Entwicklungspolitik (DIE).

Badr-Goetz, Nadja. 2007. Das Dialogische Lernmodell. Grundlagen und Erfahrungen zur Einführung einer komplexen didaktischen Innovation in den gymnasialen Unterricht. München: Meidenbauer.

Blömeke, Sigrid. 2005. «Medienpädagogische Kompetenz. Theoretische Grundlagen und erste empirische Befunde» In Kompetenzdiagnostik - Theorien und Methoden zur Erfassung und Bewertung von beruflichen Kompetenzen, hrsg. v. Andreas Frey, Reinhold S. Jäger und Ursula Renold, 76-97. Landau: Empirische Pädagogik (Berufspädagogik; 5)

Bräuer, Gerd. 2007. Beispiel für die sprachlichen Ebenen der reflexiven Praxis. https://www.phfreiburg.de/fileadmin/dateien/zentral/zwh/hochschuldidaktik/Studienportfolio/Beispiel_ für_die_sprachlichen_Ebenen_der_reflexiven_Praxis.pdf.

Dewey, John. (1910)1951. Wie wir denken. Zürich: Morgarten

Evans, Chris. 2008. «The effectiveness of $m$-learning in the form of podcast revision lectures in higher education». Computers \& Education 50(2): 491-498. https://doi.org/10.1016/j.compedu.2007.09.016.

Gibbs, Graham. 1988. Learning by Doing: A guide to teaching and learning methods. Further Education Unit, Oxford: Oxford Polytechnic. http://gdn.glos.ac.uk/gibbs/index.htm.

Hilzensauer, Wolf. 2008. «Theoretische Zugänge und Methoden zur Reflexion des Lernens. Ein Diskussionsbeitrag». Bildungsforschung.Schwerpunkt „Reflexives Lernen“ $5(2)$, hrsg. v. Thomas Häcker, Wolf Hilzensauer, und Gabi Reinmann. https://doi.org/10.25539/bildungsforschun.v2i0.77.

Hofhues, Sandra, und Tamara Bianco. 2009. «Podcasts als Motor partizipativer Hochschulentwicklung: der Augsburger „KaffeePod“». In E-Learning 2009. Lernen im Digitalen Zeitalter. Medien in der Wissenschaft Bd. 51, hrsg. v. Nicolas Apostolopoulos, Harriet Hoffmann, Veronika Mansmann, und Andreas Schwill, 235-245. Münster: Waxmann.

Kolb, David A. 1984. Experiential Learning. New Jersey:Prentice Hall.

Lonn, Steven, und Stephanie. D. Teasley. 2009. «Podcasting in higher education: What are the implications for teaching and learning?». The Internet and Higher Education 12(2): 88-92. https://doi.org/10.1016/j.iheduc.2009.06.002. 
Oswald, Kristin. 2018. Mit wissenschaftlichen Podcasts Geschichte erzählen. Aber wer hört zu? [Billet]. https://kristinoswald.hypotheses.org/2081.

Reinmann, Gabi, Silvia Hartung, und Alexander Florian. 2013. Akademische Medienkompetenz im Schnittfeld von Lehren, Lernen, Forschen und Verwalten. http://gabi-reinmann.de/wpcontent/uploads/2013/07/AkademischeMedienkompetenz_Reinmann_Hartung_Florian. pdf.

Ruf, Urs. 2008. «Das Dialogische Lernmodell». In Besser lernen im Dialog. Dialogisches Lernen in der Unterrichtspraxis, hrsg. v. Urs Ruf, Stefan Keller und Felix Winter, 13-23. Selze: Friedrich Verlag.

Tulodziecki, Gerhard. 2012. "Medienpädagogische Kompetenz und Standards in der Lehrerbildung» In Jahrbuch Medienpädagogik 9, hrsg. v. Renate Schulz-Zander, Birgit Eickelmann, Heinz Moser, Horst Niesyto und Petra Grell, 271-297. Berlin: Springer VS. https://doi. org/10.1007/978-3-531-94219-3_13.

Wedekind, Joachim. 2009. "Akademische Medienkompetenz». Schriftfassung der Virtuellen Ringvorlesung e-teaching.org vom 19.01.2009. https://www.e-teaching.org/projekt/personal/medienkompetenz/Medienkompetenz_JW.pdf.

\section{Episodenliste}

Lukács, Bence, und Constanze Reder. 2017a. «Episode 1: Über die OERlabs». (August 8). https://oerlabs.de/1-ueber-die-oerlabs/.

Andrasch, Matthias, und Bence Lukács. 2017. «Episode 5: Eine Reise durch die OER-Projektwelt». (September 6). https://oerlabs.de/episode-5-eine-reise-durch-die-oer-projektwelt/.

Lukacs, Bence. 2017. «Episode 9: Planung des Multistakeholder-Dialogs: Utopisches Denken». (November 15). https://oerlabs.de/episode-9-planung-des-multistakeholder-dialogs-utopisches-denken/.

Lukács, Bence, und Constanze Reder. 2017b. «Episode 10: Der Multistakeholder-Dialog aus Sicht der TU Kaiserslautern». (November 20). https://oerlabs.de/episode-10-der-multistakeholder-dialog-aus-sicht-der-tu-kaiserslautern/.

Andrasch, Matthias, und Constanze Reder. 2017. «Episode 12: We have a Lift-Off (MSD1 Köln)». (Dezember 20). https://oerlabs.de/episode-12-we-have-a-lift-off/.

Andrasch, Matthias, und Bence Lukács. 2018. «Episode 14: Wie läuft ein Lehramtsstudium in Köln ab?». (Januar 24). https://oerlabs.de/episode-14-wie-laeuft-ein-lehramtsstudium-inkoeln-ab/.

Andrasch, Matthias. 2018. «Episode 15: OERlabs-Studierende über ihr Spielbrett-Projekt in Kaiserslautern». (Januar 29). https://oerlabs.de/episode-15-oerlabs-studierende-in-kaiserslautern-ueber-ihr-projekt/. 